\title{
Long-term follow-up after percutaneous closure of patent foramen ovale with Amplatzer PFO Occluder: a single center experience
}

\author{
Aleksander Araszkiewicz, Marek Grygier, Sylwia Iwańczyk, Olga Trojnarska, Maciej Lesiak, Stefan Grajek \\ $1^{\text {st }}$ Department of Cardiology, Poznan University of Medical Sciences, Poznan, Poland
}

Adv Interv Cardiol 2016; 12, 1 (43): 49-54

DOI: $10.5114 /$ pwki.2016.56949

\begin{abstract}
A bstract
Introduction: Patent foramen ovale (PFO) is associated with the occurrence of cryptogenic strokes, transient neurological ischemic attacks (TIA) and migraine. Therefore despite the recent ambiguous results of prospective controlled trials, percutaneous closure of PFO is still performed in many centers.

Aim: To evaluate the safety and effectiveness of percutaneous PFO closure in the prevention of recurrence of neurologic events and migraine symptoms in long-term observation.

Material and methods: In 70 patients ( 31 male, age: $38 \pm 18$ years) percutaneous PFO closure was successfully performed with the Amplatzer PFO Occluder. An interview in conjunction with neurological follow-up was performed in all patients to reveal recurrence of embolism such as TIA or stroke and the presence of migraine symptoms before and after the procedure.

Results: The mean follow-up period was $857 \pm 363$ days (median: 571 days). No serious complications of the procedure and no death were observed. Neurological events recurred in 5 (7.1\%) patients - stroke in 1 patient, TIA in 3 patients, ocular embolism in 1 patient. All of these patients were over 40 years old or had other cardiovascular risk factors. Migraine was observed in 21 (30\%) patients before the procedure and in $11(15.7 \%)$ after the procedure $(p=0.04)$.

Conclusions: The complication rate and recurrence of neurological events in young patients after PFO closure are low. However, careful qualification and postprocedural monitoring of the patients are necessary. Patent foramen ovale closure seems to result in attenuation of migraine symptoms.
\end{abstract}

Key words: migraine, patent foramen ovale, cryptogenic stroke, prevention.

\section{Introduction}

The patent foramen ovale (PFO) is a remnant of the foramen ovale, a connection between the right and left atrium, which is an integral part of the fetal circulation. It usually closes during the first year of life. However, according to autopsy studies, PFO can be detected in 20$28 \%$ of normal hearts in adults $[1,2]$.

The prevalence of a right-to-left shunt, as a manifestation of $\mathrm{PFO}$, is high in patients with cryptogenic stroke, transient ischemic attacks (TIA) and migraine [3, 4]. Moreover, in some patients the presence of PFO may result in platypnea-orthodeoxia syndrome and provoked exercise desaturation with worse exercise capacity. Young individuals who have suffered a cryptogenic stroke associated with PFO are at risk of recurrent stroke, despite being on medication. The incidence of recurrence of stroke in these patients varies from 0 to $15 \%$ per year $[5,6]$. Currently, the treatment of these patients is controversial, although observational data that favor percutaneous PFO closure exist. Therefore, these procedures are still performed in many cardiovascular centers, despite the recent ambiguous results of prospective controlled trials of percutaneous PFO closure [7-9].

\section{Aim}

The purpose of this study was to evaluate the safety and effectiveness of percutaneous PFO closure in the prevention of recurrence of neurologic events and the number of migraine attacks or their prevalence in long-term observation.

\section{Corresponding author:}

Aleksander Araszkiewicz, MD, PhD, $1^{\text {st }}$ Department of Cardiology, Poznan University of Medical Sciences, 1/2 Długa St, 61-848 Poznan, Poland, phone: +48 6185492 93, fax: +48 6185490 94, e-mail: aaraszkiewicz@interia.pl

Received: 18.06 .2015 , accepted: 16.08 .2015$. 


\section{Material and methods}

\section{Study population}

Eighty-two patients referred to our institution with cryptogenic ischemic stroke or TIA diagnosed during 2007-2012 were evaluated. In 6 patients, the procedure was not performed because the shunt was undetected in clinical and echocardiographic evaluation. Moreover, a further 6 patients were excluded from PFO closure because of comorbid conditions. In 70 patients with confirmed PFO, percutaneous PFO closure was subsequently performed. The mean age of the patients was 38 (from 20 to 62 years, median 39 years). Clinical status of the study population and the frequency of cerebrovascular events that led to the intervention are summarized in Tables I and II, respectively.

Transient ischemic attack was defined as an acute focal neurological deficit with full recovery within $24 \mathrm{~h}$

Table I. Baseline clinical characteristics of patients $(n=70)$ in whom PFO closure was performed

\begin{tabular}{lc}
\hline Characteristics & Number (percent) \\
\hline Gender (female) & $39(55.7)$ \\
\hline Hypertension & $23(32.9)$ \\
\hline Heart disease & $2(2.9)$ \\
\hline Atrial fibrillation & $0(0)$ \\
\hline Hypercholesterolemia & $35(50.0)$ \\
\hline Diabetes & $1(1.4)$ \\
\hline Obesity & $12(17.1)$ \\
\hline Internal carotid artery stenosis & $0(0)$ \\
\hline Smoking & $17(24.3)$ \\
\hline Alcoholism & $0(0)$ \\
\hline Obstructive sleep apnea & $4(5.7)$ \\
\hline Thrombophilia & $1(1.4)$ \\
\hline Hyperhomocysteinemia & $1(1.4)$ \\
\hline Migraine & $21(30.0)$ \\
\hline Headache & $13.6)$ \\
\hline
\end{tabular}

Table II. Cerebrovascular events in patients qualified for PFO closure

\begin{tabular}{lc}
\hline Characteristic & Number (percent) \\
\hline Ischemic stroke & $56(80.0)$ \\
\hline Transient ischemic attack (TIA) & $8(11.4)$ \\
\hline Ocular vascular thrombotic event & $1(1.4)$ \\
\hline$>1$ TIA & $4(5.7)$ \\
\hline$>1$ ischemic stroke & $4(5.7)$
\end{tabular}

with or without a corresponding lesion on the computed tomography (CT) or magnetic resonance imaging (MRI) scans. Ischemic stroke referred to an acute, permanent focal neurological deficit with a corresponding destructive lesion in CT or MRI scan. Hypertension was diagnosed when blood pressure values exceeded $140 / 90 \mathrm{~mm} \mathrm{Hg}$ or when on blood pressure lowering medication. Significant (50\%) internal carotid artery stenosis was excluded in Doppler ultrasound examination. All patients had a complete neurological evaluation, biochemical workup, transthoracic echocardiography (TTE) and trans-oesophageal echocardiography (TOE). Electrocardiogram (ECG) and 24-h Holter ECG monitoring in order to reveal atrial fibrillation or other arrhythmias were performed. The whole group was evaluated for a left heart source of embolism by TTE and TOE.

Decisively, it was excluded in all patients.

\section{Migraine definition}

The diagnosis of migraine was established according to the International Headache Society criteria [10].

\section{Detection of PFO}

Transthoracic echocardiography and transoesophageal echocardiography (TOE) with saline contrast injection were used to detect PFO. The contrast injection was performed while imaging the heart with a TOE probe. Contrast passage was assessed before, during and after the Valsalva maneuver.

A PFO was judged to be present if any microbubble was seen in the left-sided cardiac chambers within 3 cardiac cycles from the maximum right atrial opacification.

\section{Inclusion and exclusion criteria for PFO closure}

A thromboembolic event was considered to be due to paradoxical embolism when the following criteria were met: presence of PFO with spontaneous or provokable right-to-left shunt confirmed in contrast TOE, ischemic stroke and/or TIA verified clinically and neuroradiologically, exclusion of any identifiable cause for the cerebrovascular ischemic events other than the PFO.

Moreover, exclusion criteria included the following: presence of other potential risk factors of thromboembolic events (e.g. the source of thrombosis within the left heart), age over 65 years, congestive heart failure, infectious endocarditis, heart valve prosthesis, internal carotid artery stenosis, thrombophilia, atrial fibrillation, intolerance of medication required during the procedure or post-procedurally, lack of patient's consent.

\section{Technique of transcatheter closure of PFO}

All patients received a loading dose of aspirin (300 mg p.o.) and clopidogrel (300 mg p.o.) and $1.0 \mathrm{~g}$ cefazolin intravenously before the procedure. During device 
implantation 5000 IU of unfractionated heparin were administered. After the procedure all the patients were treated with $75 \mathrm{mg}$ aspirin for 6 months and clopidogrel $75 \mathrm{mg}$ for 3 months. Infective endocarditis prophylaxis was recommended for 6 months.

Venous access was gained via the right femoral vein under local anesthesia with $2 \%$ lidocaine. The TOE was used during the procedure. Device implantation was guided by fluoroscopy and TOE. After identification and crossing the PFO from the right to left atrium with a 6 $\mathrm{Fr}$ multipurpose catheter a stiff guidewire was placed in the upper left pulmonary vein. Then a $10 \mathrm{Fr}$ vascular sheath was introduced. The device (Amplatzer PFO Occluder, St. Jude Medical, USA) was subsequently implanted. The size of the device was chosen depending on body size and weight, left atrial size, atrial septal anatomy, and the distance of the defect to the mitral valve and aortic sinus.

Transthoracic echocardiography was performed on the day after the procedure to confirm the correct position of the device. Additionally, in the assessment of periprocedural complications blood count was taken and 12-lead ECG recorded for over $48 \mathrm{~h}$.

\section{Follow-up evaluation}

Patients were followed for a mean of $857 \pm 363$ days (median 571 days). A structured telephone interview in conjunction with neurological follow-up was performed to reveal recurrence of embolism such as TIA and stroke, as well as migraine symptoms.

\section{Statistical analysis}

Continuous variables are expressed as mean or median and categorical data are reported as frequencies and percentages. Comparisons between groups were performed using unpaired Student's t-test or the Mann-Whitney $U$-test for continuous variables and the $\chi^{2}$ or Fisher's exact test for categorical variables. A value of $p<0.05$ was considered statistically significant.

\section{Results}

Percutaneous PFO closure was successful in all 70 (100\%) patients. During the 48-hour periprocedural period no major complications (death, tamponade, device embolization, serious bleeding) were observed. In $6(8.6 \%)$ patients minor complications were recorded. Supraventricular arrhythmias were noted in 3 (4.3\%) patients including supraventricular tachycardia in 1 (1.4\%) patient, atrial fibrillation in 1 patient and continuous supraventricular bigeminy also in 1 patient. Atrial fibrillation and frequent supraventricular extrasystole (supraventricular bigeminy) reverted spontaneously to sinus rhythm within $12 \mathrm{~h}$ after the procedure. Supraventricular tachycardia reverted with $6 \mathrm{mg}$ of adenosine intravenously and the patient was subsequently referred for electrophysiological study and ablation. In 3 (4.3\%) patients groin hematoma was observed without any serious clinical sequelae (no blood transfusion or red blood count decrease).

During the observation period, no death was encountered. In 5 (7.1\%) patients recurrent neurological events were reported: 1 stroke with permanent neurological deficit, 3 TIA and 1 ocular embolism. The first event, an ischemic stroke, occurred 5 months, and the latest event 72 months, after device implantation. Three of the 5 recurrent neurological events occurred on concomitant antiplatelet treatment, while 2 events were observed in patients on no active drug treatment (Table III). All of the patients with the exception of one, with a recurrence, had at least one cardiovascular risk factor. All patients were over 40 years old. In all these patients TOE examination excluded right-to-left shunt as a potential cause of cerebrovascular event. During follow-up, each of them was found to have one of the following: atrial fibrillation (3 patients), bileaflet aortic valve (1) and hyperhomocysteinemia (1).

The prevalence of migraine (with and without aura) was statistically significantly less frequent after the procedure. Complete cessation of migraine was achieved in $52.4 \%$ of patients with migraine before the procedure. A statistically significant and clinically important reduction in headache (which did not meet criteria of migraine) was also achieved. The reduction was 53.8\% (Table IV).

\section{Discussion}

The presence of PFO has been reported in up to $61 \%$ of patients younger than 55 years of age with cryptogenic stroke. Moreover, data from observational studies suggest an association between cryptogenic stroke and PFO [2-5]. Furthermore, the presence of PFO is associated with a 3 -fold increased risk of recurrent stroke [1-5]. There are several hypotheses explaining this phenomenon. Paradoxical embolism seems to be the most probable mechanism, but other possible explanations are formation and release of thrombus from the PFO tunnel

Table III. Cerebrovascular events in long-term follow-up in studied group

\begin{tabular}{lc} 
Characteristic & Number (percent) \\
\hline Death & $0(0)$ \\
\hline Ischemic stroke & $1(1.4)$ \\
\hline TIA & $3(4.3)$ \\
\hline Ocular embolism & $1(1.4)$ \\
\hline$>1$ TIA & $0(0)$ \\
\hline$>1$ ischemic stroke & $0(0)$ \\
\hline Migraine & $11(15.7)$ \\
\hline Headache & $7(10)$
\end{tabular}


Table IV. Prevalence of migraine and non-migraine headaches before and after PFO closure

\begin{tabular}{lccc} 
Characteristic & Before PFO closure, $n(\%)$ & After PFO closure, $n(\%)$ & Value of $p$ \\
\hline Migraine & $21(30)$ & $11(15.7)$ & 0.02 \\
\hline With aura & $4(5.7)$ & $2(2.86)$ & NS \\
\hline Without aura & $17(24.3)$ & $9(12.86)$ & NS \\
\hline Headache & $14(20)$ & $7(10)$ & 0.046
\end{tabular}

or passage of vasoactive humoral substances normally degraded in the pulmonary circulation [11-13]. Therefore it has been postulated that PFO closure should result in a decreased risk of recurrent neurologic events. Numerous observational studies have suggested that PFO closure is associated with a benefit $[14,15]$. Moreover, the safety and effectiveness of this procedure have improved dramatically over the last decade. However, a randomized trial, CLOSURE I, failed to show the superiority of closure with the STARFlex device over medical therapy alone [7]. In observational studies, the Amplatzer PFO Occluder has been shown to have advantageous safety features as a closure device [16]. However, two randomized clinical trials published recently did not confirm the effectiveness of percutaneous PFO closure with the Amplatzer device in the prevention of recurrence of cerebrovascular events. In the Randomized Evaluation of recurrent Stroke comparing PFO to Established Current standard of care Treatment (RESPECT) trial, 980 patients were randomized to closure or medical therapy groups [8]. The rate of recurrent stroke was 0.66 per 100 patient years in the closure group vs. 1.33 per 100 patient years in the medical therapy group. In the Percutaneous Treatment of Foramen Ovale versus Medical Treatment in Patients with Cryptogenic Embolism (PC) Trial for the 414 patients enrolled, the primary endpoint of death, stroke, TIA or peripheral embolism was $3.6 \%$ in the closure group and $5.2 \%$ in the medical therapy group (hazard ratio: 0.20 ; 95\% Cl: $0.02-1.72 ; p=0.14$ ) [9]. However, using the RESPECT trial definition of stroke, there was a trend toward a reduction in recurrent strokes in device closure group $(p=0.07)$. Moreover, in the RESPECT trial, in an as-treated analysis there was a significant stroke risk reduction with device closure. In young patients with cryptogenic stroke or TIA, the "attributable risk" of PFO is likely increased, and PFO closure may be of greater benefit for these patients.

The occurrence of recurrent neurological events in our group was unfortunately relatively high (7.1\%). The majority of these incidents (3 of 5) were TIA. All of them occurred in patients over 40 years of age, with risk factors that emerged after the procedure, despite careful selection of patients for PFO closure. The presence of other thromboembolic risk factors may result in the recurrence of neurologic events despite safe and effective procedure. This points to the need of very careful qualification and identification of patients who might benefit from PFO closure. After the procedure one should constantly monitor the patients and treat them in case of occurrence of thromboembolic risk factors such as atrial fibrillation. Serious complications directly associated with the procedure occurred very rarely in our study group. Supraventricular arrhythmias were temporary or were successfully treated (including RF ablation in 1 patient with atrio-ventricular nodal reentry tachycardia (AVNRT)). The percutaneous PFO closure procedure seems to be safe, effective and rarely associated with the occurrence of serious complications.

In our study migraine was observed in $30 \%$ of patients with PFO. Additionally, non-migraine headaches were reported in $18.6 \%$ of those. In our group, similarly to the previous report of Kumor et al., we found a lower incidence of migraine and non-migraine headaches after the procedure of PFO closure [17].

Despite the common prevalence of migraine, our knowledge about both pathophysiology and optimal control of symptoms remains severely limited [18]. Therefore, the data on the potential association between migraine and PFO focused our attention on the procedure of PFO closure as an opportunity of treatment.

A connection between PFO and migraine was surmised when individuals who had a right-to-left shunt closed for other reasons noted an improvement in the frequency and severity of migraine headaches [3, 4]. Research studies reported that the prevalence of right-toleft shunt is higher in patients with migraine, particularly migraine with aura $[3,17]$. Del Sette et al. and Anzola et al. determined a right-to-left shunt in patients with migraine by using transcranial Doppler ultrasound [18, 19]. The right-to-left shunt was found twice as often in patients with migraine with aura (41-48\%) as compared to those without aura (23\%) [18]. Moreover, Wilmshurst and Nightingale reported that the prevalence of migraine is higher in patients with right-to-left shunt (48\%) compared to those without a shunt (14\%) [20]. There are also retrospective studies and randomized controlled clinical trials which report a connection between migraine and the higher prevalence of ischemic stroke. Kurth et al. in the Women's Health Study validated a higher risk of ischemic stroke in women with migraine with aura especially at the age of 45 to 55 [21]. Furthermore, the meta-analysis of Etminan et al. also revealed higher prevalence of 
ischemic stroke in patients with migraine without aura [22]. However, evidence to support an overall increased frequency of PFO in migraineurs was not found. The PFO closure resulted in partial or complete relief of migraine symptoms in several retrospective single-center studies [23-26]. These findings must be interpreted cautiously, because of the weakness of the evidence. The closure of PFO in patients with frequent and intractable migraine with aura was compared to sham intervention in the only randomized, multicentre and double blind MIST trial [27]. This well-publicized and controversial study failed to show a clear benefit from device closure in individuals with particularly severe migraine. Presently, several other trials are in progress but currently there is no evidence to support device closure of PFO in migraineurs.

The presence of PFO in some patients may also lead to platypnea-orthodeoxia syndrome as well as provoked exercise desaturation with worse exercise capacity [28, 29]. Devendra et al. observed that PFO closure resulted in dramatic resolution of arterial oxygen desaturation and significant improvement in provoked exercise desaturation patients in 3-month follow-up [30]. The assessment of the exercise capacity and provoked exercise desaturation could become in future an important element of the selection of candidates for PFO closure.

\section{Conclusions}

Although the role of PFO in the etiology of cryptogenic stroke and migraine is still controversial, young patients with cryptogenic stroke and some with migraine seem to benefit from PFO closure. The PFO closure seems to be reasonable in the prevention of recurrent neurologic events in young individuals. Although the procedure is safe and associated with a low rate of early complications, careful selection of potential candidates for PFO closure is mandatory.

\section{Conflicts of interest}

The authors declare no conflict of interest.

\section{References}

1. Hagen PT, Scholz DG, Edwards WD. Incidence and size of patent foramen ovale during the first 10 decades of life: an autopsy study of 965 normal hearts. Mayo Clin Proc 1984; 59: 17-20.

2. Johansson MC, Eriksson P, Dellborg M. The significance of patent foramen ovale: a current review of associated conditions and treatment. Int J Cardiol 2009; 134: 17-24.

3. Tepper SJ, Cleves C, Taylor FR. Patent foramen ovale and migraine: association, causation, and implications of clinical trials. Curr Pain Headache Rep 2009; 13: 221-6.

4. Post MC, Budts W. The relationship between migraine and rightto-left shunt. Fact or fiction? Chest 2006; 3: 896-901.

5. Kumor M. Patent foramen ovale: to close or not to close - still the question. Postep Kardiol Inter 2012; 8: 42-7.

6. Spies C, Strasheim R, Timmermanns I, et al. Patent foramen ovale closure in patients with cryptogenic thromboembolic events using the Cardia PFO occluder. Eur Heart 2006; 27: 365-71.
7. Furlan AJ, Reisman M, Massaro J, et al.; the CLOSURE I Investigators. Closure or medical therapy for cryptogenic stroke with patent foramen ovale. N Engl J Med 2012; 366: 991-9.

8. Carroll JD, Saver JL, Thaler DE, et al.; the RESPECT Investigators. Closure of patent foramen ovale versus medical therapy after cryptogenic stroke. N Engl J Med 2013; 368: 1092-100.

9. Meier B, Kalesan B, Mattle HP, et al.; the PC Trial Investigators. Percutaneous closure of patent foramen ovale in cryptogenic embolism. N Engl J Med 2013; 368: 1083-91.

10. Headache Classification Subcommittee of the International Headache Society (2004) The International classification of headache disorders. 2nd ed. Cephalalgia 2004; 24 (Suppl. 1): 9-160.

11. Giardini A, Donti A. Transcatheter patent foramen ovale closure identifying the ideal candidate according to the available evidence. J Neurol 2005; 252: 1279.

12. Danzi GB, Sesana M, Capuano C, Baglini R. Percutaneous closure of patent foramen ovale: pathophysiology, indications, and technique. Neurol Sci 2003; 24: 17-9.

13. Halperin JL, Fuster V. Patent foramen ovale and recurrent stroke: another paradoxical twist. Circulation 2002; 105: 2580-2.

14. Schuchlenz HW, Weihs W, Berghold A, et al. Secondary prevention after cryptogenic cerebrovascular events in patients with patent foramen ovale. Int J Cardiol 2005; 101: 77-82.

15. Heinisch C, Bertog S, Wunderlich N, et al. Percutaneous closure of the patent foramen ovale using the HELEX Septal Occludder: acute and long-term results in 405 patients. Eurointervention 2012; 8: 717-23.

16. Schwerzmann M, Windecker S, Wahl A, et al. Percutaneous closure of patent foramen ovale: impact of device design on safety and efficacy. Heart 2004; 90: 186-90.

17. Kumor M, Biernacka EK, Demkow M, et al. "Migraine side effect" and stroke recurrence in late follow-up after transcatheter patent foramen ovale closure as secondary prevention of cryptogenic thromboembolism. Postep Kardiol Inter 2014; 10: 155-60.

18. Del Sette M, Angeli S, Leandri M, et al. Migraine with aura and right-to-left shunt on transcranial Doppler: a case-control study. Cerebrovasc Dis 1998; 8: 327-30.

19. Anzola GP, Meneghetti G, Zanferrari C, et al.; SAM Study Group. Is migraine associated with right-to-left shunt a separate disease? Results of the SAM study. Cephalalgia 2008; 28: 360-6.

20. Wilmshurst P, Nightingale S. Relationship between migraine and cardiac and pulmonary right-to-left shunts. Clin Sci 2001; 100: 215-20.

21. Kurth T, Slomke MA, Kase CS, et al. Migraine, headache, and the risk of stroke in women: a prospective study. Neurology 2005; 64: 1020-6

22. Etminan M, Takkouche B, Isorna FC, Samii A. Risk of ischaemic stroke in people with migraine: systematic review and metaanalysis of observational studies. BMJ 2005; 330: 63-7.

23. Rigatelli G. Migraine and patent foramen ovale: connecting flight or one-way ticket? Expert Rev Neurother 2008; 8: 1331-7.

24. Reisman M, Christofferson RD, Jesurum J, et al. Migraine headache relief after transcatheter closure of patent foramen ovale. J Am Coll Cardiol 2005; 45: 493-5.

25. Wilmshurst PT, Nightingale S, Walsh KP, Morrison WL. Effect on migraine of closure of cardiac right-to-left shunts to prevent recurrence of decompression illness or stroke or for haemodynamic reasons. Lancet 2000; 356: 1648-51.

26. Jesurum J, Fuller C, KimC, et al. Frequency of migraine headache relief following patent foramen ovale "closure" despite residual right-to-left shunt. Am J Cardiol 2008; 102: 916-20. 
27. Dowson A, Mullen MJ, Peatfield R, et al. Migraine Intervention with STARFlex Technology (MIST) trial: a prospective, multi-center, double-blind, sham-controlled trial to evaluate the effectiveness of patent foramen ovale closurewith STARFlex septal repair implant to resolve refractory migraine headache. Circulation 2008; 117: 1397-404.

28. Delgado G, Inglessis I, Martin-Herrero F, et al. Management of platypnea-orthodeoxia syndrome by transcatheter closure of atrial communication: hemodynamic characteristics, clinical and echocardiographic outcome. J Invasive Cardiol 2004; 16: 578-82.

29. Guérin P, Lambert V, Godart F, et al. Transcatheter closure of patent foramen ovale in patients with platypnea-orthodeoxia: results of a multicentric French registry. Cardiovasc Intervent Radiol 2005; 28: 164-8.

30. Devendra PD, Rane AA, Krasuski RA. Provoked exercise desaturation in patent foramen ovale and impact of percutaneous closure. J Am Coll Cardiol Intv 2012; 5: 416-9. 